\title{
ISOLATION OF BONE MARROW MESENCHYMAL STEM CELLS
}

\author{
Renata Aparecida de Camargo Bittencourt ${ }^{1}$, Hamilton Rosa Pereira ${ }^{2}$, Sérglo Luís Felisbino ${ }^{3}$, \\ Priscila Murador ${ }^{4}$, Ana Paula Ehrhardt de Olneira ${ }^{4}$, Elenice DefFune ${ }^{5}$
}

\section{SUMMARY}

Mesenchymal Stem Cells (MSCs) have a high ability to renew and differentiate themselves into various lineages of conjunctive tissues. This study aimed to isolate the MSCs from murine bone marrow by using two different growth media and to characterize them with immunostaining with antivimentin antibody. We used six 2week old BALB/c mice. Bone marrow was collected from mice's tibial and femoral channels and re-suspended in a final strength of 6x105 in Knockout-DMEM and high-glucose-DMEM media, supplemented by $10 \% \mathrm{FBS}$, and kept in a humidified 5\% CO2 incubator at $37^{\circ} \mathrm{C}$ for $72 \mathrm{~h}$, when non-adherent cells were removed during the change of medium. The number and density of adherent fibroblast-like colonies was greater with the Knockout-DMEM medium (within 5 days of culture) versus 10-20 days in DMEMhigh glucose to get the same cellular concentration. The cells in both groups were highly positive for antivimentin antibody, characterizing them as MSCs. Obtaining MSCs as quickly as possible is essential for cell therapy field, especially when those cells are intended to be used for the repair of tissues from mesenchymal sources.

Keywords: Stem Cells; Cell Culture; Mice; Bone Marrow; Vimentin.

\section{INTRODUCTION}

Bone marrow stromal cells, also known as mesenchymal stem cells or fibroblastic colony-forming units, are multipotent non-hematopoietic stem cells adhering to culture plates ${ }^{(1,2)}$.

Mesenchymal Stem Cells (MSC) of the bone marrow have the ability to renew and differentiate themselves into multiple lineages of conjunctive tissues, including bone, cartilage, adipose tissue, tendon, muscle, and bone marrow stroma ${ }^{(3,4)}$. Those cells have been first described by Friedenstein et al. ${ }^{(5)}$, who found that MSCs adhere to culture plates, look like in vitro fibroblasts, and build up colonies $^{(1,6)}$. Recently, MSCs have been brought to the attention of many researchers, because these cells are of great interest for treating various human diseases.

Many studies have isolated MSCs and controlled, in vitro, its differentiation into cartilaginous tissue and bone using specific growth factors, with the objective of using this new technology for repairing injured tissues of mesenchymal origin ${ }^{(7-9)}$.

The proposition of this study was to standardize the best culture medium that should be employed in order to quickly isolate MSCs and characterize them with immunostaining by the antivimentin antibody, due to our interest in differentiating them in vitro in a cartilaginous tissue, aiming to, in the future, use it for repairing injured joint cartilage.

\section{OBJECTIVES}

The objective of this study was to standardize culture medium that should be employed for a faster isolation of the MSCs and their characterization with immunostaining by antivimentin antibody.

\section{MATERIALS AND METHODS}

MSCs were collected from the bone marrow of six two-week old BALB/c mice, supplied by the Experimental Laboratory of Infectious Diseases, Botucatu Medical College - UNESP. The animals were sacrificed with a lethal dose of sodium pentobarbital. This research was approved by the Committee of Ethics in Animal Experiments (CEEA) of this university according to the protocol nr. 345.

Culture medium tested was the Knockout DMEM (Dulbecco's modified Eagle's medium) (DMEM; Catalog n 10829-018, Lot: 1209477) and high-glucose- DMEM (DMEM; Catalog n 12100046, Lot: 1181947), supplemented by $10 \%$ fetal bovine serum ([FBS] Catalog no 10270-106, Lot: 40Q3534K) and 10U/ml penicillin $\mathrm{G}, 10 \mathrm{ug} / \mathrm{ml}$ streptomycin and $25 \mathrm{mg} / \mathrm{ml}$ anphotericin B (Catalog $n^{\circ} 15240-096$, Lot: 1185890) all supplied by GIBCO $^{\circledR}$ Invitrogen Corporation.

MSCs were collected from femoral and tibial bone marrow of three mice by inserting a 26-gauge syringe at bone cavity, washing it with $10 \mathrm{ml}$ of Knock-out DMEM and high-glucose DMEM in the remaining three mice.

After centrifuging at $1200 \mathrm{rpm}$ for 10 minutes, bone marrow cells were re-suspended in $1 \mathrm{ml}$ at the corresponding culture media for cell counting and cell feasibility verification by using a Neubauer camera. The number of vital cells was determined by a technique that excludes non-vital cells stained by a trypan blue solution (GIB$\mathrm{CO}^{\circledR}$ Invitrogen Corporation), following the recommendations by Freshney ${ }^{(11)}$. For counting, $0.1 \mathrm{ml}$ of the cell suspension was used, which, in turn, had used $0.1 \mathrm{ml}$ of trypan blue and $0.8 \mathrm{ml}$ of culture

Study conducted at the Botucatu Medical College, Cell Culture Laboratory, Hemocenter - UNESP - São Paulo - Brazil.

Correspondences to: Faculdade de Medicina de Botucatu - UNESP, Hemocentro, Rubião Júnior s/n - E-mail: renatabit@zipmail.com.br

1 - PhD student, Department of Surgery and Orthopaedics.

2 - PhD Assistant Professor, Department of Surgery and Orthopaedics.

3 - PhD Assistant Professor, Department of Morphology - Biosciences Institute

4 - Researcher - Hemocenter.

5 - PhD Assistant Professor, Department of Urology/ Hemocenter.

Received in: 04/07/05; approved in: 05/27/05 
medium. This cell suspension was transferred to the Neubauer camera with the aid of a Pasteur pipette and then cells were counted, excluding those showing blue staining (non-feasible cells). For calculating the number of cells, the following equation was used: $\mathrm{NC} \times \mathrm{D} \times 10^{4} / \# \mathrm{Q}$, where $\mathrm{NC}=$ number of counted vital cells; $\mathrm{D}=$ sample dilution (10) and $\# Q=$ number of squares at the Neubauer camera used for counting cells. Feasibility was always higher than $95 \%$, and the number of plated cells was $6 \times 10^{5} /$ plate.

A coverslip was placed inside culture plate to allow cells to grow over it for subsequent analysis with antivimentin antibody. Cultures were maintained in a heater at $37^{\circ} \mathrm{C}$ with $5 \% \mathrm{CO}^{2}$ for $72 \mathrm{~h}$. After $72 \mathrm{~h}$ of culture, medium was refreshed in an interval of three to four days. When cells reached $80 \%$ confluence, presenting many fibroblastic colonies on the plate, coverslips were removed from culture and cells were fixed with $70 \%$ ethanol and washed twice with $2 \mathrm{ml}$ phosphate buffer saline (PBS), pH 7.4. Cultivated coverslips were treated with $3 \%$ hydrogen peroxide in methanol in order to block endogenous peroxidases. Then, they were sequentially incubated with $3 \%$ bovine serum albumin (BSA) (Sigma Chemical Co., St. Louis MO, USA). In PBS, and the primary mouse antivimentin antibody (Santa Cruz Biotech, Santa Cruz CA, USA). Subsequently, coverslips were incubated with goat IgG antimouse antibody conjugated with peroxidase (Sigma Chemical Co., St. Louis $\mathrm{MO}$, USA). Immunoperoxidase response was revealed with diaminobenzidine.

The mean area occupied by mesenchymal cells on plates was determined by the measurement of 10 fields randomly with a $5 X$ magnification, from a slide of each donor mouse. Measurements were taken by using digital images, with an image analyzer Leica $\mathrm{Q}$-win software Version 3 for Windows ${ }^{\mathrm{TM}}$.

Values achieved were expressed as average \pm standard deviation. The non-paired Student's t-test was used, of which results with a significan-

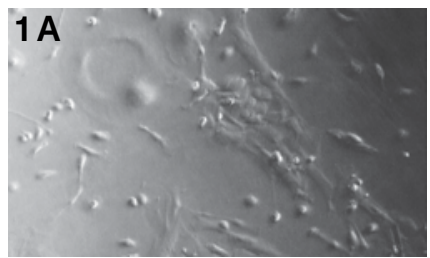

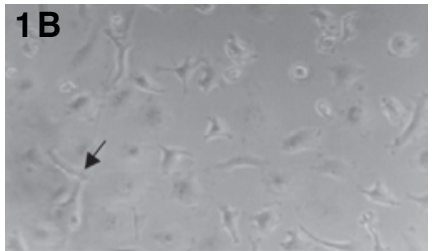
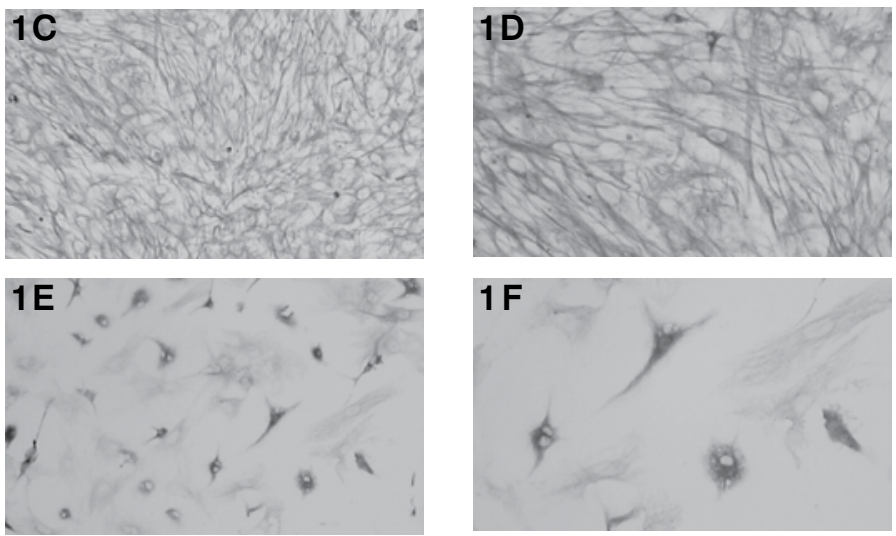

Figure 1- Phase contrast photomicrography of mesenchymal cells in culture (A-B). Presence of cell colonies with fibroblastoid morphology after 5 days in culture with the Knockout DMEM medium (A) and in high-glucose DMEM medium, cells formed colonies within 10 days $(B)$. Magnification: $x 400$ respectively. Photomicrography of mesenchymal stem cells stained by antivimentin antibody (C-F). Notice the great cellular density with elongated morphology cultured in Knockout DMEM medium $(C, D)$. Cell concentration was lower in the high-glucose DMEM medium (E, F). Magnification: (C, E) x200 (D, F) x400.

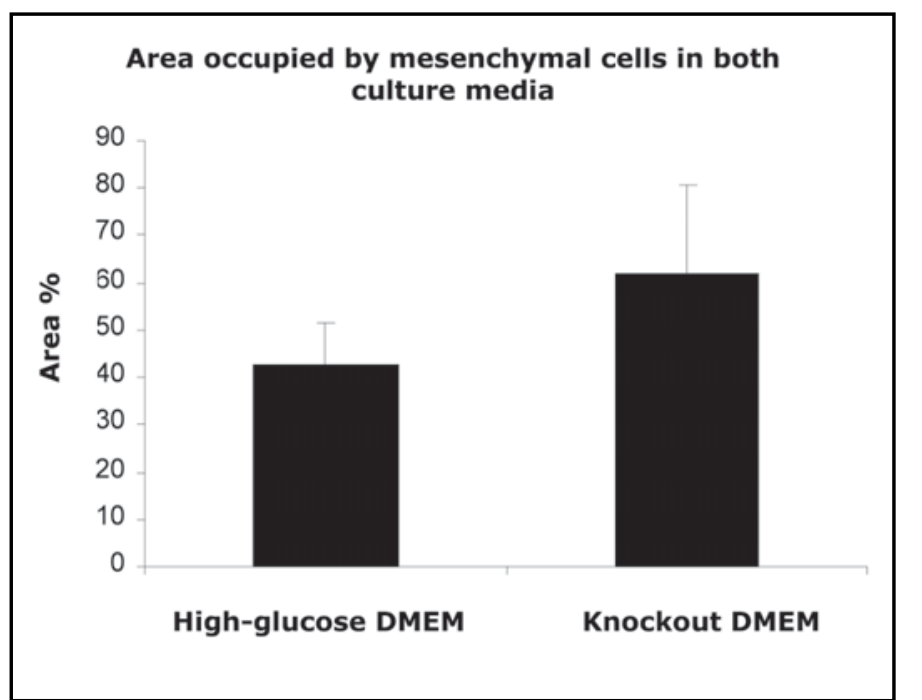

Figure 2 - Comparison of the effects between both culture media on the area occupied by mesenchymal cells. Values represent the average \pm standard deviation. Cells cultured in Knockout DMEM medium occupied a plate area approximately $50 \%$ larger than those cultured in high-glucose DMEM medium, showing a statistically significant difference, $p<0.01$. ce level $(p \leq 0.05)$ were considered as significant. Statistical test was

\section{RESULTS}

Colonies of cells with a fibroblastoid morphology started to appear on culture plates in 72 hours with the Knockout DMEM medium se DMEM. Non-adherent cells were removed from culture during medium refreshment. After 5 days of culture, the number and density of colonies of fibroblastoid-like cells with the Knockout DMEM medium (Figure 1a) were greater than with the high-glucose DMEM, which took nearly 10 days to reach the same cell concentration of the first medium (Figure 1b). Cells maintained in culture in both protocols were positive to antivimentin, characterizing them as MSCs. After 10 days, culture plate containing the Knockout DMEM medium was thoroughly covered by MSCs, as confirmed by antivimentin antibody response (Figure 1c and 1d). However, the plate where the high-glucose DMEM medium was used did not present the same cell strength ( $\mathrm{Fi}$ gure $1 \mathrm{e}$ and $1 \mathrm{f}$ ).

The mean area occupied by mesenchymal cells as analyzed by morphometry (Figure 2) was approximately $61.89 \%$, with standard deviation of 18.64 for Knockout DMEM versus $42.88 \%$, with standard deviation of 8.81 for high-glucose DMEM, with $p<0.01$, showing a statistically significant result.

\section{DISCUSSION}

Results show that it is possible to obtain MSCs in a short time by using the Knockout DMEM culture medium. This was possible due to its ability to be used specifically in embryonic stem cells. In other studies, the culture of MSCs with low-glucose DMEM was performed, and the authors achieved the first adherent fibroblastoid-like ceIls colonies only after 5 days $^{(4,10)}$

In this study, we observed a significant difference between 
both culture media, and those differences were more evident when cells were stained with the antivimentin antibody.

\section{CONCLUSIONS}

1. The use of both media enabled to show the superiority of Knockout DMEM, which produced a large number of cells when compared to the amounts achieved when the high-glucose DMEM was used.
2. The use of the Knockout DMEM medium could be an alternative for a quicker isolation of the MSCs in culture, especially when it is desired to differentiate them in other mesenchymal lineage tissues, such as cartilage.

\section{Acknowledgements:}

We acknowledge the technician Mrs. Fátima Regina Guimarães, from the Cell Culture Laboratory, Hemocenter - Botucatu Medical College - UNESP, for her priceless assistance.

\section{REFERENCES}

1. Javason EH, Colter DC, Schwarz EJ, Prockop DJ. Rat marrow stroma cells are more sensitive to plating density and expand more rapidly from single-cell-derived colonies than human marrow stromal cells. Stem Cells 2001; 19:219-25.

2. Hung SC, Chen NJ, Hsieh SL, Li H, Ma HL, Lo WH. Isolation and characterization of size-sieved stem cells from human bone marrow. Stem Cells 2002; 20:249-58.

3. Campagnoli C, Roberts AG, Kumar S, Bennett PR, Bellantuonol, Fisk NM Identification of mesenchymal stem/progenitor cells in human first-trimester fetal blood, liver, and bone marrow. Blood 2001; 98:2396-402.

4. Romanov YA, Svintsitskaya VA, Smirnov VN. Searching for alternative sources of postnatal human mesenchymal stem cells: candidate MSC-like Cells from umbilical cord. Stem Cells 2003; 21:105-10.

5. Friedenstein AJ, Deriglazova UF, Kulagina NN Panasuk AF, Rudakowa SF, Luria EA, Ruadkow IA. Precursors for fibroblasts in different populations of hematopoietic cells as detected by the in vitro colony assay method. Exp Hematol 1974; 2:83-
6. Johnstone B. Mesenchymal stem cells and condrogenesis. Eur Cells Mater 2002: 4 (Suppl I):1473-2262

7. Martin I, Padera R F, Vunjak-Novakovic G, Freed LE. In vitro differentiation of chick embryo bone marrow stroma cells into cartilaginous and bone-like tissues. J Orthop Res 1998; 16:181-9.

8. Meirelles LS, Nardi NB. Murine marrow-derived mesenchymal stem cell: isolation, in vitro expansion, and characterization. Br J Hematol 2003, 123:702-11.

9. Pittenger MF, Mackay AM, Beck SC, Jaiswal RK, Douglas R, Mosca JD et al. Multilineage potencial of adult human mesenchymal stem cells. Science 1999; 284:143-7.

10. Lee HS, Huang GT, Chiang H, Chiou LL, Chen MH, Hsieh CH, Jiang CC. Multipotential mesenchymal stem cells from femoral bone marrow near the site of osteonecrosis. Stem Cells 2003; 21:190-9.

11. Freshney RI. Culture of animal cells: a manual of basic technique. $3^{\text {rd }}$. Ed. New York: Wiley-Liss, 2001 